Article

\title{
Assessing the Fauna Diversity of Marudu Bay Mangrove Forest, Sabah, Malaysia, for Future Conservation
}

\section{Mohamed Zakaria ${ }^{1, *}$ and Muhammad Nawaz Rajpar ${ }^{2}$}

1 Faculty of Forestry, Universiti Putra Malaysia, UPM International, Serdang 43400, Selangor Darul Ehsan, Malaysia; E-Mail: mzakaria@upm.edu.my

2 Sindh Wildlife Department, Opposite PIA Reservation Office, Moulana Din Muhammad Road, Saddar, Karachi 77550, Pakistan; E-Mail: rajparnawaz@gmail.com

* Author to whom correspondence should be addressed; E-Mail: mzakaria@upm.edu.my; Tel.: +60-192-690-355; Fax: +60-389-432-514.

Academic Editor: Peter Saenger

Received: 24 February 2015 / Accepted: 21 April 2015 / Published: 30 April 2015

\begin{abstract}
Mangrove is an evergreen, salt tolerant plant community, which grows in inter-tidal coastal zones of tropical and subtropical regions of the world. They are ecologically important for many fauna species and are rich in food resources and consist of many different vegetation structures. They serve as ideal foraging and nursery grounds for a wide array of species such as birds, mammals, reptiles, fishes and aquatic invertebrates. In spite of their crucial role, around $50 \%$ of mangrove habitats have been lost and degraded in the past two decades. The fauna diversity of mangrove habitat at Marudu Bay, Sabah, East Malaysia was examined using various methods: i.e. aquatic invertebrates by swap nets, fish by angling rods and cast nets, reptiles, birds, and mammals through direct sighting. The result showed that Marudu Bay mangrove habitats harbored a diversity of fauna species including 22 aquatic invertebrate species (encompassing 11 crustacean species, six mollusk species and four worm species), 36 fish species, 74 bird species, four reptile species, and four mammal species. The wide array of fauna species could be due to the availability of complex vegetation structures, sheltered beaches and tidal mudflats, which are rich in food resources and also offer safe foraging and breeding grounds for them. These heterogeneous habitats must be protected in a sustainable way in order to ensure the continued presence of aquatic and terrestrial fauna species for future generations.
\end{abstract}

Keywords: fauna; habitat; food; mangrove; Marudu Bay 


\section{Introduction}

Mangrove is a salt tolerant plant that occurs within inter-tidal zones where a river enters into the sea, especially in the coastal belt [1,2]. They are a highly productive, tropical coastal ecosystem encompassing estuaries, creeks, lagoons, backwaters, mud-flats, salt-pans and islands which has both great aquatic and terrestrial biodiversity [3] and plays an integral role in coastal ecosystem functions at the interface between terrestrial, freshwater and marine systems [4]. Mangrove fauna are animal communities that inhabit or utilize mangrove habitats, such as coastal inter-tidal zones, estuaries and riverine areas where rivers drain into the sea, to fulfill their need for survival and reproduction $[5,6]$. The vegetation structure and composition of mangrove areas may vary depending upon soil texture and structure, rainfall pattern, and inflow of freshwater from rivers to the sea [7,2].

Mangrove forests are considered as a highly productive ecosystem, i.e., they provide important ecological and economic services [8,9]. They play a crucial role in providing suitable habitats for fauna, safe breeding and chick rearing grounds, nurseries for a diversity of fishes and shellfishes, as well as ideal foraging grounds for animals such as fishes, birds and aquatic invertebrates and refuge from predators [10]. Mangrove habitats are consistently undervalued, degraded and are being lost at an alarming rate due to human intervention, i.e., coastal development, deforestation, tin mining, urbanization, salt production, conversion into paddy fields and aquaculture ponds, over-harvesting of timber and fuelwood, pollution, i.e., dumping of domestic sewage and crude oil exploration [2,11-14].

It has been stated that around $50 \%$ of mangrove habitats has been lost and degraded in the past two decades due to human interventions $[15,10]$. The mangrove habitat loss and degradation have posed major threats to a wide array of fauna $[16,17]$ bringing them among the ranks of endangered and extinct species [2,18-20].

Recently, considerable attention has been paid to mangrove fauna diversity and habitat due to their importance and functions [21]. There has been no detailed study undertaken to examine the fauna diversity in Marudu Bay mangrove forest, Sabah, Malaysia. Thus, there was an urgent need to examine the fauna diversity inhabiting mangrove habitats in order to understand the impact of disturbance for future conservation and management.

This current study was aimed at examining the fauna diversity (such as birds, mammals, reptiles, fishes and aquatic invertebrates) inhabiting or utilizing the downstream to upstream mangrove and river tributaries of Marudu Bay. The scientific baseline data obtained from the study would provide an in depth understanding of the current fauna diversity status for better future conservation and management initiatives.

\section{Materials and Methods}

\subsection{Study Site}

Marudu Bay mangrove forest is located between latitude $6^{\circ} 15^{\prime \prime}$ to $6^{\circ} 45^{\prime \prime} \mathrm{N}$ and a longitude of $116^{\circ}$ to $117^{\circ}$ E, $113 \mathrm{~km}$ from Kota Kinabalu Sabah, Malaysia (Figure 1). The forest is still in pristine condition, i.e., $>90 \%$ intact, spread over several creeks and lagoons and covers an area of 9550 ha. This mangrove forest is managed, in accordance with the principles of conservation and sustainable use, by the Sabah Forest Department, Malaysia. The mangrove forest consists of 16 tree species of 12 genera and nine families, such as Rhizopora apiculata, R. mucronata, Bruguiera parviflora, B. gymnorrhiza and Ceriops 
decandra. Marudu Bay mangrove forest is rich in fauna diversity, i.e., aquatic invertebrates, fishes, birds, reptiles and mammals. This mangrove forest provides a wide range of ecosystem services such as timber, fuel-wood, fisheries, eco-tourism and nursery habitat for different animals. Marudu Bay mangrove forest is an important source of livelihood for local communities.

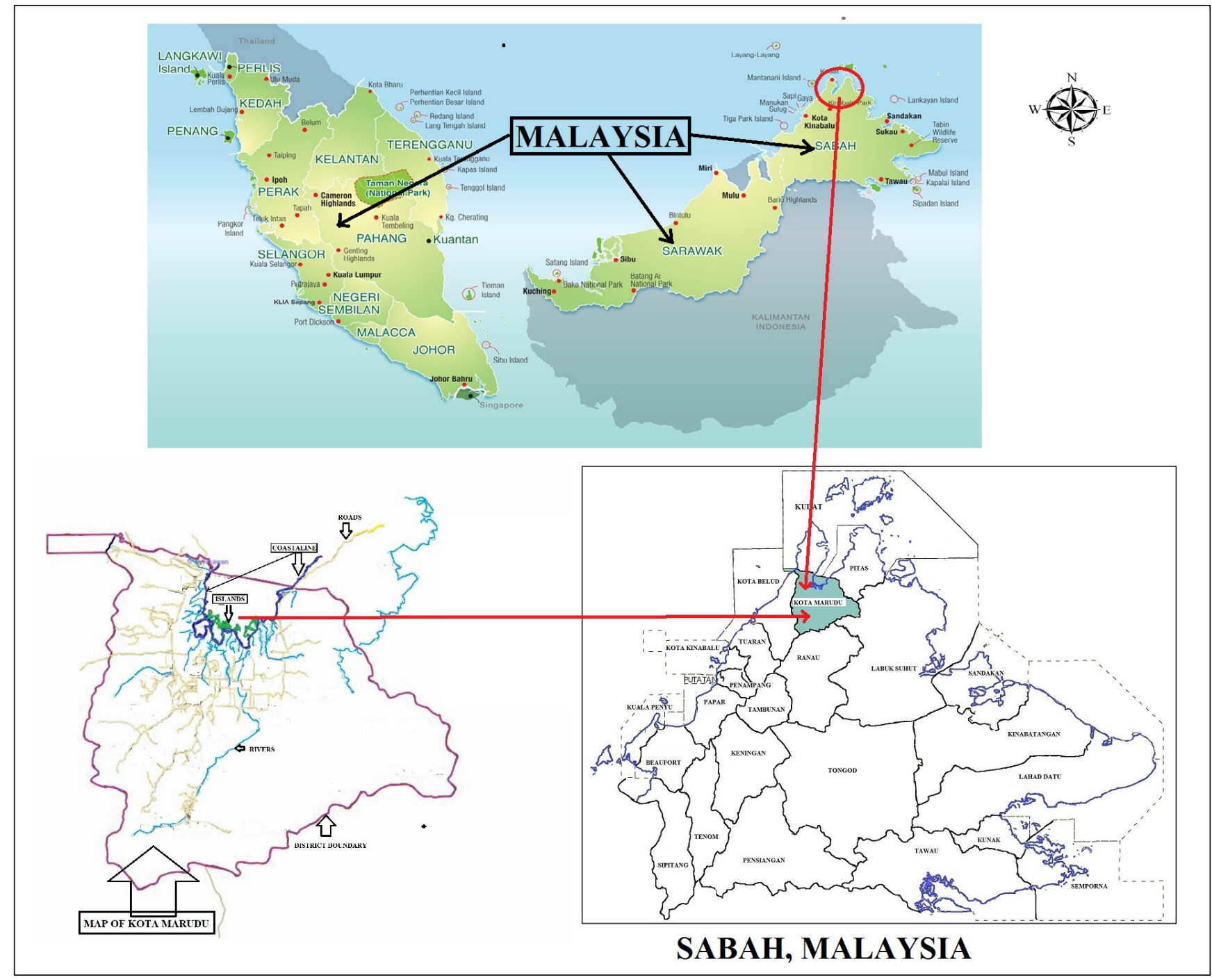

Figure 1. Location of the Marudu Bay Mangrove forest Sabah, Malaysia.

\subsection{Fauna Surveys}

The presence of birds, mammals, and reptiles was determined by boat using direct observation using $10 \times 50$ binoculars from 23 March to 3 April 2009, 21-27 May 2009 and 2-8 February 2010. A total of 60 point stations along the three rivers and mudflats was established randomly to cover different habitat types, i.e. from downstream to upstream Marudu Bay mangrove forest. The location of each point station was at intervals of $250 \mathrm{~m}$ apart. The main reason for locating point stations $250 \mathrm{~m}$ apart was to avoid double counting the same species at more than one station. This was based on the recommendation by [22] who stated this approach was suitable: (i) for dense habitats, such as forest and shrubs; (ii) to survey cryptic, shy, and skulking species; (iii) for the populations that are of higher density and are more species rich; (iv) for situations where access is restricted; and (v) particularly for bird-habitat studies. 
The survey was conducted by boat along selected streams as it was the easiest way to observe the birds in the mangrove forests early in the morning (between 6:30 a.m. and 7:00 a.m. depending on weather) and to be completed before 12:00 p.m. The methodology followed was developed by [23,24].

The fish fauna were caught through angling rods and cast nets while aquatic invertebrate were collected through swap nets.

\section{Results}

\subsection{Aquatic Invertebrate Fauna}

Marudu Bay mangrove habitats were rich in aquatic invertebrate fauna (i.e., 22 species), which comprised crustaceans (crabs, prawns and shrimps), mollusks (snails, clams, periwinkle, murex and oysters) and worms (lug worms, tube worms, eunicid worms and polychaete worms; Table 1).

Table 1. List of aquatic invertebrate species detected in Marudu Bay mangrove habitat.

\begin{tabular}{|c|c|c|}
\hline Family Name & Common Name & Scientific Name \\
\hline \multicolumn{3}{|c|}{ Crustacean Species } \\
\hline Alpheidae & Mangrove Snapping Prawns & Alpheus sp. \\
\hline Grapsidae & Mangrove Root Crab & Goniopsis cruentata \\
\hline Limulidae & Horseshoe Mangrove Crab & Tachypleus gigas \\
\hline \multirow[t]{3}{*}{ Ocypodidae } & Orange-clawed Fiddler Crab & Uca coarctata \\
\hline & Orange Fiddler Crab & Uca vocans \\
\hline & Rosy Fiddler Crab & Uca rosea \\
\hline \multirow[t]{2}{*}{ Penaeidae } & Greasy-back Shrimp & Metapenaeus ensis \\
\hline & Banana Prawn & Penaeus merguiensis \\
\hline Portunidae & Giant Mud Crab & Scylla serrata \\
\hline Sesarmidae & Mangrove Tree Crab & Aratus pisonii \\
\hline Squillidae & Silver Mantis Shrimp & Harpiosquilla harpax \\
\hline \multicolumn{3}{|c|}{ Mollusks Species } \\
\hline Cerithiidae & Cerith Snail & Cerithium $\mathrm{sp}$. \\
\hline Cyrenidae & Mud Clam & Polymesoda erosa \\
\hline Ellobiidae & Mangrove Helmet Snail & Cassidula sp. \\
\hline Lithorinidae & Mangrove Periwinkle & Littorina angulifera \\
\hline Muricidae & Mangrove Murex & Chicoreus capuncinus \\
\hline Onchidiidae & Grey-footed Mangrove Onch Slug & Platyvindex sp. \\
\hline Ostreidae & Mangrove Tree Oyster & Crassostrea sp. \\
\hline \multicolumn{3}{|c|}{ Worm Species } \\
\hline Arenicolidae & Lugworms & Arenicola cristata \\
\hline Chaetopteridae & Parchment Tube Worm & Chaetopterus sp. \\
\hline Eunicidae & Eunicid Worm & Lysidice sp. \\
\hline Onuphidae & Polychaete worms & Diopatra sp. \\
\hline
\end{tabular}

\subsection{Fish Fauna}

Angling rods and cast nets caught a total of 36 fish species belonging to 22 families from mangrove and river tributaries of the Marudu Bay habitats. The findings highlighted that Leiognathidae, Lutjanidae 
and Sciaenidae were three most abundant fish families based on the number of captured species (i.e., each three fish species) in the study area. In contrast, 12 fish families were recorded as the rarest in the study area, i.e., only one fish species was captured for each family (Table 2).

Table 2. List of fish species captured in Marudu Bay mangrove and river tributaries.

\begin{tabular}{|c|c|c|}
\hline Family Name & Common Name & Scientific Name \\
\hline \multirow{2}{*}{ Ambassidae } & Buru Glass Perchlet & Ambassis buruensis \\
\hline & Naked-head Glassy Perchlet & Ambassis gymnocephalus \\
\hline Apogonidae & Hookfin Cardinalfish & Apogon griffini \\
\hline Belonidae & Spottail Needle Fish & Strongylura strongylura \\
\hline \multirow{2}{*}{ Carangidae } & Hardtail Scad & Megalaspis cordyla \\
\hline & Talang Queenfish & Scomberoides commersonnianus \\
\hline Chirocentridae & Whitefin Wolf herring & Chirocentrus nudus \\
\hline Drepaneidae & Concertina Fish & Drepane longimana \\
\hline \multirow{2}{*}{ Eleotridae } & Flatheaded Sleeper & Ophiocara porocephala \\
\hline & Tenpounder & Elops machnata \\
\hline Engraulidae & Anchovy Fish & Anchovia spp. \\
\hline \multirow{2}{*}{ Gerreidae } & Whipfin Silver-biddy & Gerres filamentosus \\
\hline & Silver Biddy & Gerres erythrourus \\
\hline \multirow{2}{*}{ Gobiidae } & Mangrove Goby & Acentrogobius caninus \\
\hline & Tank Goby & Glossogobius giuris \\
\hline \multirow{2}{*}{ Haemulidae } & Saddle Grunt & Pomadasys maculatus \\
\hline & Javelin Grunt & Pomadasys kaakan \\
\hline Hemiramphidae & Halfbeak or Garfish & Hemiramphus spp. \\
\hline \multirow{3}{*}{ Leiognathidae } & Whipfin Ponyfish & Equulites leuciscus \\
\hline & Spotnape Ponyfish & Nuchequula nuchalis \\
\hline & Shortnose Ponyfish & Leiognathus brevirostris \\
\hline \multirow{3}{*}{ Lutjanidae } & Black Snapper & Apsilus dentatus \\
\hline & One Spot Snapper & Lutjanus monostigma \\
\hline & Mangrove Red Snapper & Lutjanus argentimaculatus \\
\hline Megalopidae & Indo-Pacific Tarpon & Megalops cyprinoides \\
\hline \multirow{2}{*}{ Mugilidae } & Greenback Mullet & Liza subviridis \\
\hline & Square-tail Mullet & Liza vaigiensis \\
\hline Plotosidae & White-lipped Eel Catfish & Paraplotosus albilabris \\
\hline Scatophagidae & Spotted Scat & Scatophagus argus \\
\hline \multirow{3}{*}{ Sciaenidae } & Goatee Croaker & Dendrophysa russelii \\
\hline & Orange Spotted Grouper & Epinephelus coioides \\
\hline & Cloudy Grouper Fish & Epinephelus erythrurus \\
\hline \multirow{2}{*}{ Siganidae } & Streaked Spinefoot Fish & Siganus javus \\
\hline & Goldlined Spinefoot & Siganus guttatus \\
\hline Terapontidae & Jarbua Terapon & Terapon jarbua \\
\hline Trichiuridae & Large-head Hairtail & Trichiurus lepturus \\
\hline
\end{tabular}

Very few reptile species were detected during the study. The reptile fauna comprised of crocodiles, monitor lizards and skinks (Table 3). 
Table 3. List of reptile species detected at Marudu Bay mangrove river tributaries.

\begin{tabular}{lll}
\hline \multicolumn{1}{c}{ Family Name } & \multicolumn{1}{c}{ Common Name } & \multicolumn{1}{c}{ Scientific Name } \\
\hline Crocodylidae & Crocodile & Crocodylus palustris \\
\hline \multirow{2}{*}{ Varanidae } & Mangrove Monitor Lizard & Varanus indicus \\
& Malaysian Water Monitor Lizard & Varanus salvator \\
\hline Scincidae & Mangrove Skink & Emoia atrocostata \\
\hline
\end{tabular}

\subsection{Bird Fauna}

Bird fauna of downstream to upstream mangrove and river tributaries included 74 species representing 33 families. The results showed that Ardeidae (nine bird species), Scolopacidae (six species), Cuculidae and Picidae (each five species) were the four most dominant families in Marudu Bay mangrove habitats. On the contrary, 17 families, i.e., Anhingidae, Apodidae, Artamidae, Campephagidae, Chloropseidae, Ciconiidae, Coraciidae, Dicaeidae, Hemiprocnidae, Hirundinidae, Muscicapidae, Pachycephalidae, Psittacidae, Rhipiduridae, Sittidae, Timaliidae, and Sternidae were the rarest in the study area (i.e., only one species detected for each family. Out of all bird species, six species were endangered, one rare and one nearly threatened (Table 4).

Table 4. List of bird species detected in Marudu Bay Mangrove habitat.

\begin{tabular}{|c|c|c|}
\hline Family Name & Common Name & Scientific Name \\
\hline \multirow{3}{*}{ Accipitridae } & Brahminy Kite & Haliastur indus \\
\hline & White-bellied Fish-Eagle & Haliaeetus leucogaster \\
\hline & Osprey & Pandion haliaetus \\
\hline \multirow{4}{*}{ Alcedinidae } & Stork-billed Kingfisher & Pelargopsis capensis \\
\hline & Common Kingfisher & Alcedo atthis \\
\hline & Collared Kingfisher & Halcyon chloris \\
\hline & White-throated Kingfisher & Halcyon smymensis \\
\hline Anhingidae & Oriental Darter (E) & Anhinga melanogaster \\
\hline Apodidae & White-bellied Swiftlet & Collocalia esculenta \\
\hline \multirow{9}{*}{ Ardeidae } & Little Egret & Egretta garzetta \\
\hline & Little Heron & Butorides striatus \\
\hline & Great Egret & Casmerodius albus \\
\hline & Intermediate Egret & Egretta intermedia \\
\hline & Chinese Egret (E) & Egretta eulophotes \\
\hline & Cattle Egret & Bubulcus ibis \\
\hline & Schrenck's Bittern & Ixobrychus eurhythmus \\
\hline & Yellow Bittern & Ixobrychus sinensis \\
\hline & Rufous Night-Heron & Nycticorax caledonicus \\
\hline Artamidae & White-breasted Wood-Swallow & Artamus leucorhynchus \\
\hline Campephagidae & Pied Thriller & Lalage nigra \\
\hline \multirow{3}{*}{ Charadriidae } & Pacific Golden-Plover & Pluvialis fulva \\
\hline & Little Ringed Plover & Charadrius dubius \\
\hline & Common Ringed Plover & Charadrius hiaticula \\
\hline Chloropseidae & Common Iora & Aegithina tiphia \\
\hline Ciconiidae & Lesser Adjutant (E) & Leptoptilos javanicus \\
\hline
\end{tabular}


Table 4. Cont.

\begin{tabular}{|c|c|c|}
\hline Family Name & Common Name & Scientific Name \\
\hline \multirow{3}{*}{ Columbidae } & Pink-necked Green Pigeon & Treron vernans \\
\hline & Little Green-Pigeon & Treron olax \\
\hline & Jambu Fruit-Dove & Ptilinopus jambu \\
\hline Coraciidae & Dollar Bird & Eurystomus orientalis \\
\hline \multirow{5}{*}{ Cuculidae } & Black-bellied Malkoha (E) & Phaenicophaeus diardi \\
\hline & Chestnut-bellied Malkoha (E) & Phaenicophaeus sumatranus \\
\hline & Greater Coucal & Centropus sinensis \\
\hline & Malayan Bronze Cuckoo & Chrysococcyx minutillus \\
\hline & Lesser Coucal & Centropus bengalensis \\
\hline Dicaeidae & Scarlet-backed Flowerpecker & Dicaeum curentatum \\
\hline \multirow{2}{*}{ Estrilidae } & Dusky Munia & Lonchura fuscans \\
\hline & Black-headed Munia & Lonchura articapilla \\
\hline Hemiprocnidae & Whiskered Treeswift & Hemiprocne comata \\
\hline Hirundinidae & Pacific Swallow & Hirundo tahitica \\
\hline \multirow{2}{*}{ Laniidae } & Bar-winged Flycatcher-Shrike & Hemipus picatus \\
\hline & Black-winged Flycatcher-Shrike & Hemipus hirundinaceus \\
\hline \multirow{2}{*}{ Meropidae } & Blue-tailed Bee-eater & Merops philippinus \\
\hline & Blue-throated Bee-eater & Merops viridus \\
\hline Muscicapidae & Mangrove Blue-Flycatcher & Cyornis rufigastra \\
\hline \multirow{4}{*}{ Nectariniidae } & Olive-backed Sunbird & Nectarinia jugularis \\
\hline & Plain Sunbird & Anthreptes simplex \\
\hline & Brown-throated Sunbird & Anthreptes malacensis \\
\hline & Purple-throated Sunbird & Nectarinia sperata \\
\hline Pachycephalidae & Mangrove Whistler & Pachycephala grisola \\
\hline \multirow{2}{*}{ Phalacrocoracidae } & Great Cormorant (R/A) & Phalacrocorax carbo \\
\hline & Little Cormorant & Phalacrocorax niger \\
\hline \multirow{5}{*}{ Picidae } & Common Flameback & Dinopium javanense \\
\hline & Brown-capped Woodpecker & Picoides moluccensis \\
\hline & Speckled Piculet & Picumnus innominatus \\
\hline & White-bellied Woodpecker & Dryocopus javensis \\
\hline & Maroon Woodpecker & Blythipicus rubiginosus \\
\hline Psittacidae & Blue-crowned Hanging Parrot & Loriculus galgulus \\
\hline Rhipiduridae & Pied Fantail & Rhipidura javanica \\
\hline \multirow{6}{*}{ Scolopacidae } & Common Redshank & Tringa totanus \\
\hline & Nordmann's Greenshank (E) & Tringa guttifer \\
\hline & Grey-tailed Tattler (NT) & Tringa brevipes \\
\hline & Common Sandpiper & Tringa hypoleucos \\
\hline & Terek Sandpiper & Xenus cinereus \\
\hline & Whimbrel & Numenius phaeopus \\
\hline Sittidae & Velvet-fronted Nuthatch & Sitta frontalis \\
\hline \multirow{2}{*}{ Sturnidae } & Philippine Glossy Starling & Aplonis panayensis \\
\hline & Hill Myna & Gracula religiosa \\
\hline
\end{tabular}


Table 4. Cont.

\begin{tabular}{lll}
\hline \multicolumn{1}{c}{ Family Name } & \multicolumn{1}{c}{ Common Name } & \multicolumn{1}{c}{ Scientific Name } \\
\hline \multirow{3}{*}{ Sylviidae } & Ashy Tailorbird & Orthotomus ruficeps \\
& Common Tailobird & Orthotomus sutorius \\
& Rufous-tailed Tailorbird & Orthotomus sericeus \\
\hline Timaliidae & Striped Tit-Babbler & Macronus gularis \\
\hline \multirow{2}{*}{ Turdidae } & Magpie Robin & Copsychus saularis \\
& White-rumped Shama & Copsychus malabaricus \\
\hline Sternidae & Whiskered Tern & Childonias hybrida \\
\hline & $\mathrm{NT}=$ Nearly Threatened; R/A = Rare/Accidental; E = Endangered.
\end{tabular}

\subsection{Mammal Fauna}

Only three mammal species belonging to three families were detected during the study period, which include monkeys, pigs and squirrels (Table 5).

Table 5. List of mammal species detected at Marudu Bay mangrove river tributaries.

\begin{tabular}{lll}
\hline \multicolumn{1}{c}{ Family Name } & \multicolumn{1}{c}{ Common Name } & \multicolumn{1}{c}{ Scientific Name } \\
\hline \multirow{2}{*}{ Cerocopithecidae } & Long-tailed Macaque & Macaca fascicularis \\
& Dusky-leaf Monkey & Trachypithecus obscurus \\
\hline Suidae & Wild Pigs & Sus scrofa \\
\hline Sciuridae & Plantain Squirrel & Callosciurus notatus \\
\hline
\end{tabular}

\section{Discussion}

The recording of a higher diversity of avian, fish, reptile, mammal and aquatic invertebrate species illustrated that Marudu Bay mangrove and river tributary habitats had attracted a wide array of fauna species. It has been stated that mangrove habitats may harbor a wide range of animals such as birds, mammals, reptiles, fishes, and aquatic invertebrates [25-28]. The presence of a higher diversity of fauna could be due to the habitat's pristine condition (i.e., no disturbance), complex vegetation structure and composition [29,30], the availability and richness of food resources such as fish, polychaetes, mollusks, crabs, and crustaceans [31-33] and low predation risk [25]. The vegetation structure and composition, occurrence of mudflat and richness of food resources are the major driving factors that influence the distribution and diversity of animals directly or indirectly. Vegetation heterogeneity abundance of food resources and habitat diversity may increase avian richness and diversity [34,35]; i.e. they provide suitable foraging and chick rearing grounds, and protection from harsh weather and predators [2].

The recording of a higher number of fish species illustrated that Marudu Bay mangrove areas serve as a nursery ground for various juvenile fish communities [36-40]. It may be that these areas are rich in invertebrate assemblages such as crustaceans (crabs, prawns and shrimps), mollusks (snails, clams, periwinkle, murex and oysters) and worms (lugworms, tube worms, eunicid worms and polychaete worms). In addition, the extensive root systems of mangroves create habitat heterogeneity and complexity, offering suitable foraging sites for juvenile fishes and protecting them from predators by reducing their visibility [32,33]. Habitat heterogeneity and complexity is a major factor that influences fauna diversity and distribution [6,41-43]. 
The sampling of a higher number of aquatic invertebrate species-i.e., 22 species including crustacean, mollusk, periwinkle, murex and worm species - indicated that the Marudu Bay mangrove and river tributary habitats are rich in organic food material that created suitable habitats for them. The presence of the high diversity of invertebrates could also be due to the availability of sheltered beaches and tidal mudflats, which are rich in organic matter. It has been stated that aquatic invertebrates such as periwinkle inhabit the bottom of mudflats (periwinkle-Tympanotonus fuscatus, oysters-Crassostrea sp., mangrove crab-Goniopsis sp., mud crab-Panopeus sp., land crab-Cardiosoma sp.) [44]. Mangrove aquatic invertebrates are a major dietary component of birds, fishes and even other invertebrates.

The recording of sufficient numbers of threatened, nearly threatened and rare bird species such as Oriental Darter, Chinese Egret, Lesser Adjutant, Black-bellied Malkoha, Nordmann's Greenshank, and Grey-tailed Tattler shows that Marudu Bay mangrove forest is potentially vital habitat for a wide array of avian species. These avian species utilize these areas as an important wintering ground, i.e., they fulfill their daily requirements such as habitat, water and food and also protection from predators and harsh weather. These endangered avian species has become threatened from habitat loss and destruction due to conversion of mangrove areas into oil palm plantation and coastal development. The habitat loss and degradation has seriously affected on their population. For their continuous survival and existence, they need special attention for protection and conservation.

\section{Conclusion}

Based the results of this study, it is concluded that Marudu Bay mangrove forest, Sabah, Malaysia, must be protected in a sustainable way in order to protect its diverse aquatic and terrestrial fauna species for future generations.

\section{Author Contributions}

Mohamed Zakaria Reviewed and edited the manuscript.

Muhammad Nawaz Rajpar Developed the experimental design, Collected and analyzed the data and wrote the manuscript.

\section{Conflicts of Interest}

The authors declare no conflict of interest.

\section{References}

1. Zhou, Y.; Zhao, B.; Peng, Y.; Chen, G. Influence of mangrove reforestation on heavy metal accumulation and speciation in intertidal sediments. Mar. Pollut. Bull. 2010, 6, 1319-1324.

2. Rajpar, M.N.; Zakaria, M. Mangrove Fauna of Asia. In Mangrove Ecosystems of Asia, Status, Challenges and Management Strategies; Hanum, F., Mohamad, A.L., Hakeem, K.R., Ozturk, M., Eds.; Springer Science + Business Media: New York, NY, USA, 2014; p. 500.

3. Mmom, C.P.; Arokoyu, S.B. Mangrove forest depletion, biodiversity loss and traditional resources management practices in the Niger Delta, Nigeria. Res. J. Appl. Sci. Eng. Technol. 2010, 2, $28-34$. 
4. Spalding, M.; Kainuma, M.; Collins, L. World Atlas of Mangroves; Earthscan: London, UK; Washington, DC, USA, 2010.

5. Wan Juliana, W.A.; Damanhuri, A.; Razali, M.S.; Norhayati, A.; Latiff, A. Mangrove Flora of Langkawi; Langkawi Research Centre, Institute for Environment and Development (LESTRI), Universiti Kebagsaan Malaysia, and Langkawi Development (LDA): Bangi Selangor, Malaysia, 2010; p. 150.

6. Tayefeh, F.H.; Zakaria, M.; de Marchi, G.; Amini, H.; Moradi, A.; Ahmadpour, P.; Ghasemi, S. Breeding biology of the Crab Plover (Dromas ardeola) on the Mond Islands, Northern Persian Gulf, Iran. Waterbirds 2013, 36, 448-462.

7. Rotaquio, E.L., Jr.; Nakagoshi, N.; Rotaquio, R.L. Species composition of mangrove forests in Aurora, Philippines-A special reference to the presence of Kandelia Candel (L.) Druce. J. Int. Dev. Coop. 2007, 13, 61-78.

8. Wagner, G.M.; Akwilapo, F.D.; Mrosso, S.; Ulomi, S.; Masinde, R. Assessment of Marine Biodiversity, Ecosystem Health, and Resource Status in Mangrove Forests in Mnazi Bay Ruvuma Estuary Marine Park; Nairobi: IUCN EARO, 2004; p. 106.

9. Pawar, P.R. Species diversity of birds in mangroves of Uran (Raigad), Navi Mumbai, Maharashtra, West coast of India. J. Exp. Sci. 2011, 2, 73-77.

10. Ellison, J.C. Climate Change Vulnerability Assessment and Adaptation Planning for Mangrove Systems; World Wildlife Fund (WWF): Washington, DC, USA, 2012; p. 142.

11. Macintosh, D.J.; Ashton, E.C.; Havanon, S. Mangrove rehabilitation and intertidal biodiversity: A study in the Ranong Mangrove ecosystem, Thailand. Estuar. Coast. Shelf Sci. 2002, 55, 331-345.

12. Duke, N.C.; Meynecke, J.O.; Dittmann, S.; Ellison, A.M.; Anger, K.; Berger, U.; Cannicci, S.; Dielle, K.; Ewel, K.C.; Field, C.D.; et al. A world without mangrove? Science 2007, 317, 41-42.

13. Gracia, S.M.; Rosenberg, A.A. Food security and marine capture fisheries: Characteristics, trends, drivers and future perspective. Philos. Trans. R. Soc. Lond. Biol. Sci. 2010, 365, 2869-2880.

14. Baba, S.; Chan, H.; Aksornkoae, S. Useful Products from Mangrove and Other Coastal Plants; ISME Mangrove Educational Book Series No. 3; International Society for Mangrove Ecosystems (ISME), Okinawa, Japan; International Tropical Timber Organization (ITTO), Yokohama, Japan, 2013.

15. Giri, C.; Ochieng, E.; Tieszen, L.L.; Zhu, Z.; Singh, A.; Loveland, T.; Masek, J.; Duke, N. Status and distribution of mangrove forests of the world using Earth observation satellite data. Glob. Ecol. Biogeogr. 2011, 20, 154-159.

16. Henle, K.; Davies, K.F.; Kleyer, M.; Margules, C.; Settele, J. Predictors of species sensitivity to fragmentation. Biodivers. Conserv. 2004, 13, 207-251.

17. Ewers, R.M.; Didham, R.K. Confounding factors in detection of species responses to habitat fragmentation. Biol. Rev. Camb. Philos. Soc. 2006, 81, 117-142.

18. Sandilyan, S.; Thiyagesan, K.; Nagarajan, R. Major decline in species-richness of waterbirds in the Pichavaram mangrove wetlands, southern India. Wader Study Group Bull. 2010, 117, 91-98.

19. Penha-lopes, G.; Torres, P.; Cannicci, S.; Narciso, L.; Paula, J. Monitoring anthropogenic sewage pollution on mangrove creeks in southern Mozambique: A test of Palaemon concinnus Dana, 1852 (Palaemonidae) as a biological indicator. Environ. Pollut. 2011, 159, 636-645. 
20. Zhigang, Y.; Ermi, Z.; Haitao, S.; Diesmos, A.; Alcala, A.; Brown, R.; Afuang, L.; Gee, G.; Sukumaran, J., Yaakob, N.; et al. Fejervarya cancrivora. In IUCN 2012; IUCN Red List of Threatened Species. Version 2012.2; IUCN, 2004.

21. Macintosh, D.J.; Ashton, E.C. A review of mangrove biodiversity conservation and management. In Center for Tropical Ecosystem; Final Report; University of Aarhus: Aarhus, Denmark, 2002; p. 86.

22. Gregory, R.D.; Gibbons, D.W.; Donald, P.F. Bird census and survey techniques. In Bird Ecology and Conservation; A Handbook of Techniques; Sutherland, W.J., Newton, I., Green, R.E., Eds.; Oxford University Press: Oxford, UK, 2006; pp. 17-55.

23. Richardson, A.J.; Taylor, I.R.; Growns, J.E. The foraging ecology of egrets in rice fields in southern New South Whales, Australia. Waterbirds 2001, 24, 255-264.

24. Yu-Seong, C.; Kwon, I.; Yoo, J. A study of feeding methods in five species of heron and egrets in Korea. J. Field Biol. 2008, 31, 147-151.

25. Almany, G.R. Does increased habitat complexity reduce predation and competition in coral reef fish assemblages? Oikos 2004, 106, 275-284.

26. Weis, S.J. Use of international mangrove and sea wall habitats by coral reef fishes in the Wakatobi marine park, Indonesia. Raffles Bull. Zool. 2005, 53, 119-124.

27. Talaat, W.A.W.; Tahir, N.M.; Husain, M.L. Mangrove Biodiversity Management and Conservation in Malaysia: The Present Legislative, Administrative and Policy Framework. In Proceeding of Universiti Malaysia Terengganu (UMTAS 2011). International annual symposium on life science, Kuala Terengganu, Malaysia, 11-13 July 2011; pp. 399-404.

28. Nyanti, L.; Ling, T.Y.; Jongkar, G. Fish and Crustacean Communities and Fish Length-Weight Relationship of Lutong River, Miri, Sarawak, Malaysia. World J. Fish Mar. Sci. 2012, 4, 102-110.

29. Gratwicke, B.; Speight, M.R. The relationship between fish species richness, abundance and habitat complexity in a range of shallow tropical marine habitats. J. Fish Biol. 2005, 66, 650-667.

30. Fuchs, T. Effects of habitat complexity on invertebrate biodiversity. Immed. Sci. Ecol. 2013, 2, $1-10$.

31. Verweij, M.C.; Nagelkerken, I.; Wartenbergh, S.L.J.; Pen, I.R.; van der Velde, G. Caribbean mangroves and seagrass beds as daytime feeding habitats for juvenile French grunts, Haemulon flavolineatum. Mar. Biol. 2006, 149, 1291-1299.

32. Correa, D.A.; de Oliveira, M. Composition of the aquatic invertebrate fauna associated to the mangrove vegetation of a coastal river, analysed through a manipulative experiment. Pan-Am. J. Aquat. Sci. 2008, 3, 23-31.

33. Wang, M.; Huang, Z.; Shi, F.; Wang, W. Are vegetated areas of mangroves attractive to juvenile and small fish? The case of Dongzhaigang Bay, Hainan Island, China. Estuar. Coast. Shelf Sci. 2009, 85, 208-216.

34. Malavasi, R.; Battisti, C.; Carpaneto, G.M. Seasonal changes in bird assemblages of a remnant wetland in a Mediterranean landscape: Implications for management. Ornis Hung. 2008, 17-18, $25-33$.

35. Kissling, W.D.; Sekercioglu, C.H.; Jetz, W. Bird dietary guild richness across latitudes, environments and biogeographic regions. Glob. Ecol. Biogeogr. 2012, 21, 328-340. 
36. Chittaro, P.M.; Usseglio, P.; Sale, P. Variation in fish density, assemblage composition and relative rates of predation among mangrove, seagrass and coral reef habitats. Environ. Biol. Fishes 2005, 72, 175-187.

37. Aguilar-Perera, A.; Appeldoorn, R.S. Variation in juvenile fish density along the mangrove-sea grass coral reef continuum in SW Puerto Rico. Mar. Ecol. Prog. 2007, 348, 139-148.

38. Nakamura, Y.; Horinouchi, M.; Shibuno, T.; Tanaka, Y.; Miyajima, T.; Koike, I.; Kurokura, H.; Sano, M. Evidence of ontogenetic migration from mangroves to coral reefs by black-tail snapper Lutjanus fulvus: Stable isotope approach. Mar. Ecol. Progr. 2008, 355, 257-266.

39. Jaxion-Harm, J.; Saunders, J.; Speight, M.R. Distribution of fish in seagrass, mangroves and coral reefs: Life-stage dependent habitat use in Honduras. Int. J. Trop. Biol. 2012, 60, 683-698.

40. Dehghani, M. Study of fish mangrove communities and comparison of traditional fisheries methods in Hara Biosphere Reserve. Mar. Biodivers. Rec. 2014, 7, e107.

41. Saha, N.; Aditya, G.; Saha, G.K. Habitat complexity reduces prey vulnerability: An experimental analysis using aquatic insect predators and immature dipteran prey. J. Asia-Pac. Entomol. 2009, 12, 233-239.

42. Firstater, F.N.; Hidalgo, F.J.; Lomovasky, B.J.; Ramos, E.; Gamero, P.; Iribarne, O.O. Habitat structure is more important than important than nutrient supply in modifying mussel bed assemblage in an upwelling area of the Peruvian coast. Helgol. Mar. Res. 2011, 65, 187-196.

43. Kovalenko, K.E.; Thomaz, S.M.; Warfe, D.M. Habitat complexity: Approaches and future directions. Hydrobiologia 2012, 685, 1-17.

44. Omogoriola, H.O.; Williams, A.B.; Ukaonu, S.C.; Adegbile, O.M.; Olakolu, F.C.; Mbawuike, B.C.; Akinnigbagbe, A.E.; Ajulo, A.A. Survey, biodiversity and impacts of economic activities on mangrove ecosystem in eastern part of Lagos Lagoon, Nigeria. Nat. Sci. 2012, 10, 30-34.

(C) 2015 by the authors; licensee MDPI, Basel, Switzerland. This article is an open access article distributed under the terms and conditions of the Creative Commons Attribution license (http://creativecommons.org/licenses/by/4.0/). 